\title{
Accuracy of intraocular lens calculation formulas for eyes with insufficient capsular support
}

\author{
Zhouyue Li ${ }^{1 \#}$, Zhangkai Lian ${ }^{1 \#}$, Charlotte Aimee Young ${ }^{2}$, Jing Zhao $^{1}$, Guangming Jin ${ }^{1}$, Danying Zheng $^{1}$ \\ ${ }^{1}$ State Key Laboratory of Ophthalmology, Zhongshan Ophthalmic Center, Sun Yat-sen University, Guangzhou, China; ${ }^{2}$ Department of \\ Ophthalmology, Third Affiliated Hospital, Nanchang University, Nanchang, China \\ Contributions: (I) Conception and design: G Jin, D Zheng; (II) Administrative support: G Jin, D Zheng; (III) Provision of study materials or patients: \\ None; (IV) Collection and assembly of data: Z Li, Z Lian; (V) Data analysis and interpretation: G Jin, Z Li, Z Lian; (VI) Manuscript writing: All \\ authors; (VII) Final approval of manuscript: All authors. \\ "These authors contributed equally to this work. \\ Correspondence to: Professor Danying Zheng. State Key Laboratory of Ophthalmology, Zhongshan Ophthalmic Center, Sun Yat-sen University, \\ Guangzhou 510060, China. Email: zhengdyy@163.com; Guangming Jin, MD. State Key Laboratory of Ophthalmology, Zhongshan Ophthalmic \\ Center, Sun Yat-sen University, Guangzhou 510060, China. Email: jingm@mail2.sysu.edu.cn.
}

Background: There is no consensus on which intraocular lens (IOL) power calculation formula provides the best refractive prediction in patients with inadequate capsular support whose anterior ocular anatomic structure differs from that of normal subjects. Therefore, the purpose of this study was to analyze the accuracy and performance of IOL calculation formulas (SRK/T, Holladay 1, Hoffer Q, Haigis, and Barrett Universal II) in predicting postoperative refractive prediction error (PE) for this subgroup of patients.

Methods: A total of 110 eyes from 110 patients with insufficient capsular support who underwent scleral fixation of an IOL at the Zhongshan Ophthalmic Center from July 1, 2016 to November 30, 2019 were enrolled in this retrospective study. Preoperative optical biometrics were measured with the IOL Master 500 (Carl Zeiss, Oberkochen, Germany). The performance of each formula in predicting PE was compared, and the effect of keratometry and axial length (AL) on PE was evaluated for each formula using univariate and multivariate linear regression analysis.

Results: The mean age of the included participants was $12.54 \pm 9.66$ years. The Sanders, Retzlaff, and Manus/theoretical (SRK/T) $(-0.25 \mathrm{D})$ and Holladay $1(-0.28 \mathrm{D})$ formulas tended to have minimal postoperative PE compared to the Hoffer Q $(-0.62 \mathrm{D})$, Haigis $(-0.67 \mathrm{D})$, and Barrett Universal II $(-0.62 \mathrm{D})$ formulas $(\mathrm{P}=0.005)$. All formulas individually resulted in $<70 \%$ of eyes within $\pm 1.00 \mathrm{D}$ of the PE. Nevertheless, after constants were optimized, these formulas led to $7.3 \%$ to $13.6 \%$ of increase within $\pm 1.00 \mathrm{D}$ of the PE. Keratometry and AL were significantly associated with PE for each formula, but the relationship was weakest for SRK/T.

Conclusions: In eyes with insufficient capsular support, postoperative PE was minimal for the SRK/T formula, which suggested SRK/T to be the best choice, especially when the keratometry and AL of patients are extremely large or small.

Keywords: Insufficient capsular support; sclera-sutured intraocular lens (sclera-sutured IOL); intraocular lens calculation (IOL calculation); accuracy; prediction refraction error

Submitted Apr 13, 2020. Accepted for publication Nov 26, 2020.

doi: 10.21037/atm-20-3290

View this article at: http://dx.doi.org/10.21037/atm-20-3290 


\section{Introduction}

The capsule of the crystalline lens is usually the assumed position for the intraocular lens (IOL) after implantation. However, some ocular comorbidities, such as ocular trauma (1), lens dislocation (2-4), and congenital or secondary weakness of zonules can result in inadequate capsular support and even cause severe visual impairment. For patients with such conditions, transscleral-fixated IOL implantation is one of the commonly used management strategies (5-7). Accurate intended surgical refractive prediction error $(\mathrm{PE})$ is crucial for patients with inadequate capsular support, especially for young children who require good visual acuity (VA) to prevent amblyopia but whose compliance with spectacles is poor.

Preoperative estimation of postoperative IOL position, postoperative refraction determination, and preoperative axial length (AL) have been identified as the main factors contributing to PE (8). The Sanders, Retzlaff, and Manus/ theoretical (SRK/T), Holladay 1, Hoffer Q, Haigis, and Barrett Universal II (Barrett II) formulas are five theoretical vergence formulas that are commonly built in optical biometers and are easily accessible online. They are based on Gaussian optics and depend on AL, keratometry, or other variables to estimate effective lens position and calculate IOL power. These IOL calculation formulas are commonly determined or optimized based on biometry parameters from common cataract-affected eyes with routine in-thebag implantation of IOL, and have demonstrated excellent predictability with similar accuracy for eyes with an AL between 22-26 mm (9). However, prediction for eyes with inadequate capsular support is still challenging.

Compared to normal eyes, patients with inadequate capsular support due to conditions such as congenital ectopia lentis often have a longer AL (10), flatter cornea $(4,11)$, and a larger variation of anterior chamber depth (ACD) measurement, all of which may present difficulties for IOL calculation. Therefore, application of the optimized IOL constants available on the User Group for Laser Interference Biometry (ULIB, http://ocusoft.de/ulib/) for preoperative IOL calculation may be not suitable for patients with inadequate capsular support. However, to our knowledge, no study to date has evaluated the accuracy of IOL calculation formulas or optimized their IOL constants for this subgroup of patients.

We conducted this study to assess and compare the accuracy of five commonly used IOL calculation formulas (SRK/T, Holladay 1, Hoffer Q, Haigis, and Barrett II) for the evaluation of postoperative PE with optimized IOL constants in patients with insufficient capsular support who underwent transscleral-fixated IOL implantation. The extent of bias within each formula for different biometric dimensions of the eye (AL and corneal curvature) was also investigated. We present the following article in accordance with the Strengthening the Reporting of Observational Studies in Epidemiology (STROBE) reporting checklist (available at http://dx.doi.org/10.21037/atm-20-3290).

\section{Methods}

This was a retrospective study of patients with insufficient capsular support who underwent lens extraction and transscleral IOL fixation at the Zhongshan Ophthalmic Center in Guangzhou, China between July 1, 2016 and November 30, 2019. All study participants had unilateral or bilateral surgery performed by the same surgeon (Dr. D Zheng). The study was conducted in accordance with the Declaration of Helsinki (as revised in 2013). The study was approved by ethical committee of Zhongshan Ophthalmic Center (NO.: 2019KYPJ184) and individual consent for this retrospective analysis was waived.

Our selection criteria were summarized as follows: (I) eyes with insufficient capsule support and accepted transscleral-fixated IOL implantation; (II) biometrics measured using the IOL Master 500 (Carl Zeiss, Oberkochen, Germany); (III) keratometric cylinder <4.0 D; and (IV) manifest refraction within the 3 to 5 weeks postoperatively. Patients with any of the following were excluded: (I) a history of corneal or fundus disease; (II) severe postoperative complications, such as uveitis or glaucoma; (III) $<6 / 20$ best corrected vision post operation or invalid biometry. If both eyes of one participant met the inclusion criteria, only the first eye to receive surgery was included in this study.

The surgical procedures were as follows: after the conjunctiva was cut, limbal-based partial thickness scleral flaps were performed at the 4 and 10 o'clock positions, with the posterior edge located $3 \mathrm{~mm}$ behind the limbus. The capsular bag was completely removed in each eye, and an IOL was transsclerally fixated at the 4 and 10 o'clock positions under the scleral flaps with 10-0 Prolene sutures (Ethicon Inc., Cornelia, Georgia, USA). Each IOL was sutured at approximately $2 \mathrm{~mm}$ posterior to the limbus. Rayner 970C and 920H IOL models (Rayner Intraocular Lenses Ltd., Worthing, West Sussex, UK) were implanted; both models share a similar aspherical design and the same 
Table 1 Demographic and biometric data of all patients

\begin{tabular}{ll}
\hline Parameters & Value \\
\hline Patients & 110 \\
Female (\%) & $44(40.0)$ \\
Age, mean \pm SD (years) & $12.54 \pm 9.66$ \\
Eyes: right/left & $62 / 48$ \\
Axial length, mean \pm SD $(\mathrm{mm})$ & $25.84 \pm 2.75$ \\
Average keratometry, mean \pm SD (D) & $41.04 \pm 1.77$ \\
$\quad$ Flat keratometry & $40.10 \pm 1.72$ \\
Steep keratometry & $41.99 \pm 1.94$ \\
ACD \pm SD (mm) & $3.46 \pm 0.69$ \\
Intraocular lens power, mean \pm SD (D) & $17.64 \pm 6.25$ \\
\hline
\end{tabular}

$\mathrm{D}$, diopter; ACD, anterior chamber depth; SD, standard deviation.

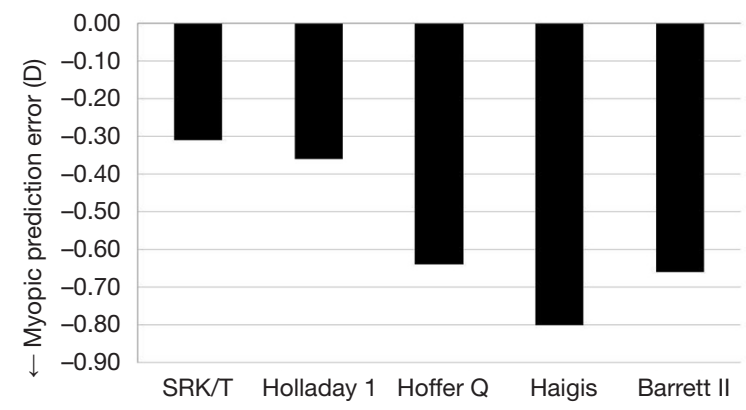

Figure 1 Comparison of PEs (in diopter) for the five formulas. Note that myopic prediction errors are indicated by negative values. PEs, prediction errors.

manufacturer's constant but have different diopter ranges.

Data of patient demographics, operative eye, preoperative optical biometrics measured with the IOL Master 500, postoperative refraction, and best corrected visual acuity (BCVA) were collected. All data mentioned above were entered in an electronic medical record by doctors and/or biometry technicians, and the authors of this study collected the data retrospectively.

Constants for the IOL formulas that we adopted postoperatively were the optimized values on the ULIB website, which were the default values of the IOL Master. We then adjusted the lens constants to reduce the mean error to zero for the SRK/T, Holladay 1, and Hoffer Q formulas using their mathematical formulas, as described in previous studies by using Microsoft Excel (Office 2019, Microsoft Corp., Redmond, WA, USA)
(12-14). Lens constants for Haigis (http://www.eyecalcs. com/WEBCALCS/IOLcalc2/IOL2.html) and Barrett II formulas (www.apacrs.org/BARRETT_UNIVERSAL2/) were optimized online.

\section{Statistical analysis}

All statistical analyses were performed using SPSS Version 16.0 (SPSS 16.0, Inc., Chicago, IL, USA). The ShapiroWilk test was performed to determine whether variables followed a normal distribution. All values are presented as mean \pm standard deviation, except if their distribution was non-normal. The postoperative PE between the five formulas was compared using one-way analysis of variance (ANOVA), with post-hoc testing on indication and correction for multiple comparisons. Comparisons between absolute errors (AEs) were performed using repeated measure ANOVA (the Friedman test with Wilcoxon signed-rank post-hoc analyses and Bonferroni correction). Univariable and multivariable linear regression analyses were used to identify the association of the postoperative PE with $\mathrm{AL}$ and keratometry. A two-tailed $\mathrm{P}$ value of $<0.05$ was considered to be statistically significant.

\section{Results}

A total of 110 eyes from 110 patients (44 females, 62 males) with implantation of Rayner $970 \mathrm{C} / 920 \mathrm{H}$ models were included in this study. The mean age of the study participants was $12.54 \pm 9.66$ years. Demographic characteristics of all participants are summarized in Table 1.

Systematic biases in refractive prediction were found in all of the formulas investigated. The mean PE for the SRK/T, Holladay 1, Hoffer Q, Haigis, and Barrett II formulas exhibited a myopic shift from the target refraction (Figure 1). The result of one-way ANOVA showed that the mean $\mathrm{PE}$ was relatively higher with Hoffer Q, Haigis, and Barrett II than with SRK/T and Holladay $1(\mathrm{P}=0.005)$. Distribution around the median AE is shown in Figure 2. The Friedman test demonstrated significant differences in median $\mathrm{AE}(\mathrm{P}=0.002)$ between the five formulas, and according to the results of post-hoc analysis, SRK/T had a lower median AE than Hoffer Q, Haigis, and Barrett II (all $\mathrm{P}<0.05)$.

Optimized lens constants are shown in Table 2. The optimized lens constants in this study were slightly smaller than the values reported on the ULIB website. The median $\mathrm{AE}$ and median PE for the ULIB constants, before and 


\section{Page 4 of 8}

after optimization, are summarized in Table 3. There was no significant difference in median $\mathrm{AE}$ between the five formulas after the lens constants were optimized $(\mathrm{P}=0.565)$. Before optimization, percentage of eyes within $\pm 1.00 \mathrm{D}$ of the PE of SRK/T, Holladay1, Hoffer Q, Haigis and Barrett II were $69.9 \%, 65.5 \%, 57.3 \%, 54.5 \%$ and $65.5 \%$, respectively. After optimization, as a contrast, percentage of eyes within $\pm 1.00 \mathrm{D}$ of the PE increase to $79.1 \%, 72.7 \%$, $68.2 \%, 68.2 \%, 74.5 \%$ respectively.

The results of univariate and multivariate linear regression analyses of the relationship of postoperative $\mathrm{PE}$ with $\mathrm{AL}$ and keratometry are summarized in Table 4. In the univariate linear regression analyses, $\mathrm{AL}$ was found to be positively associated with postoperative $\mathrm{PE}$ for each formula, but the association appeared to be weaker for SRK/ $\mathrm{T}$ and Barrett II than for the other formulas. Keratometry was also significantly associated with postoperative PE for each formula, but the association was weakest for SRK/ T. Additionally, in the multivariate linear regression analysis, the association between $\mathrm{AL}$ and postoperative

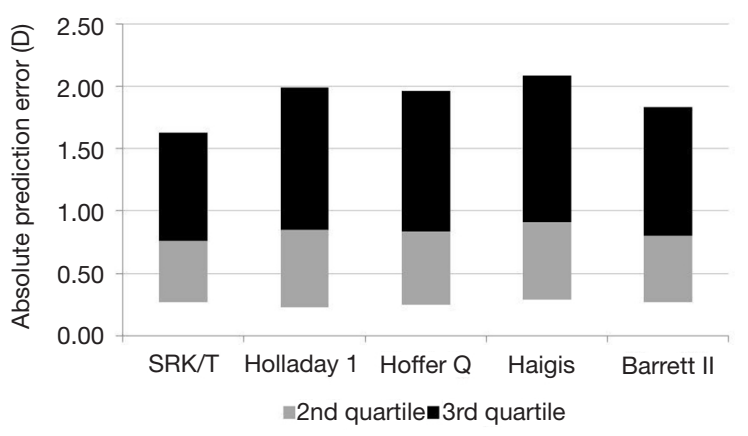

Figure 2 Box plot of the mean AEs (in diopter) for the five formulas. Gray boxes represent the second quartile, and black boxes represent the third quartile. AEs, absolute errors.

\section{Li et al. IOL calculation for eyes with insufficient capsular support}

PE was still significant for each formula after adjustment for the keratometry, and a significant association between keratometry and postoperative $\mathrm{PE}$ was observed for each formula after adjustment for the AL.

\section{Discussion}

In this study, we found that postoperative PE for scleralsutured IOL positioned $2 \mathrm{~mm}$ posterior to the limbus showed a myopic shift from the target refraction with all estimated formulas using the constants from the ULIB website. The SRK/T and Holladay 1 formulas had better outcomes than the Hoffer Q, Haigis, and Barrett formulas in terms of accuracy of postoperative PE. Additionally, the performance of SRK/T was better than that of Hoffer Q, Haigis, and Barrett based on the value of the median AE. Nevertheless, no significant difference in median AE was observed between any of the estimated formulas after optimization of the constants. This study, to our knowledge, is the first to analyze the accuracy and performance of formulas to predict postoperative refraction outcomes in patients with insufficient capsular support after lens extraction and primary transscleral fixation of an IOL.

One of the main factors influencing postoperative $\mathrm{PE}$ is the position of the IOL, which has varied in patients with scleral-fixated IOL implantation in previous studies (15-21). The most common choice of suture location in the sclera is $1.5-2 \mathrm{~mm}$ posterior to the limbus. In the current study, all scleral-fixated IOL were placed $2 \mathrm{~mm}$ posterior to the limbus, and no significant postoperative complications, such as anterior chamber cells and flare, corneal edema, elevated intraocular pressure, or wound leak, were reported in participants' follow-up medical records. For adults, a scleral-fixated IOL positioned $3 \mathrm{~mm}$ behind the limbus was assumed to be the "in-the-bag" IOL position due to the slight difference in postoperative PE from the target

Table 2 Optimized lens constants of Rayner 970C/920H compared to ULIB

\begin{tabular}{lccr}
\hline \multirow{2}{*}{ Formula } & Lens constant name & Optimized & Mean \\
\cline { 3 - 4 } SRK/T & A constant & 117.86 & 118.3 \\
Holladay 1 & Surgeon factor & 1.08 & 1.41 \\
Hoffer Q & ACD & 4.63 & 5.21 \\
Haigis & a0 (a1, a2) & $0.30(0.40,0.10)$ & $1.02(0.40,0.10)$ \\
Barrett II & Lens factor & 0.94 & 1.52 \\
\hline
\end{tabular}

ACD, anterior chamber depth; ULIB, User Group for Laser Interference Biometry. 
Table 3 The median AE and mean PE after and before optimization

\begin{tabular}{lcccc}
\hline \multirow{2}{*}{ Formula } & \multicolumn{2}{c}{ Median AE $($ IQR) in diopter } & \multicolumn{2}{c}{ Mean PE (SD) in diopter } \\
\cline { 2 - 5 } SRK/T & Optimized & ULIB & Optimized & $-0.01(0.94)$ \\
Holladay 1 & $0.50(0.27,0.87)$ & $0.56(0.22,1.09)$ & $0.00(1.04)$ & $-0.31(0.97)$ \\
Hoffer Q & $0.62(0.23,1.14)$ & $0.65(0.29,1.33)$ & $0.00(1.10)$ & $-0.36(1.11)$ \\
Haigis & $0.59(0.25,1.13)$ & $0.73(0.47,1.59)$ & $0.00(1.12)$ & $-0.64(1.21)$ \\
Barrett II & $0.62(0.29,1.18)$ & $0.88(0.46,1.71)$ & $-0.01(0.98)$ & $-0.60(1.27)$ \\
\hline
\end{tabular}

$\mathrm{AE}$, absolute error; PE, prediction error; ULIB, User Group for Laser Interference Biometry; IQR, interquartile range; SD, standard deviation.

Table 4 Results of univariable and multivariate analyses to evaluate the relationship of postoperative PE with AL and keratometry

\begin{tabular}{|c|c|c|c|c|}
\hline Formulas & \multicolumn{2}{|c|}{$\mathrm{AL}$} & \multicolumn{2}{|c|}{ Keratometry } \\
\hline $\mathrm{SRK} / \mathrm{T}$ & $0.32^{* *}$ & $0.32^{* *}$ & $0.19^{\star}$ & $0.20^{*}$ \\
\hline Holladay 1 & $0.53^{\star \star}$ & $0.54^{\star *}$ & $0.28^{\star \star}$ & $0.30^{\star \star}$ \\
\hline Hoffer Q & $0.52^{\star \star}$ & $0.53^{\star \star}$ & $0.41^{\star \star}$ & $0.43^{\star \star}$ \\
\hline Barrett II & $0.26^{\star *}$ & $0.34^{* *}$ & $0.33^{\star *}$ & $0.27^{\star \star}$ \\
\hline
\end{tabular}

${ }^{*}, \mathrm{P}<0.05 ;{ }^{* *}, \mathrm{P}<0.01 . \mathrm{PE}$, prediction error; $\mathrm{AL}$, axial length.

refraction $(-0.19 \pm 0.72 \mathrm{D})(15)$; however, little is known about the relationship between the position of scleral-fixated IOL and the "in-the-bag" IOL position in children with insufficient capsular support. It is difficult to determine the anatomic lens position by suture location in the sclera for scleral-fixated IOL; thus, the postoperative refraction following implantation of scleral-fixated IOL can vary. Our results showed that the postoperative PE was myopic for all formulas when the IOL power for a desired postoperative refraction was determined by assuming that the IOL was positioned in the capsular bag, suggesting that the location of IOL plane was in front of the "in-the-bag" position for patients with scleral-fixated IOL positioned $2 \mathrm{~mm}$ posterior to the limbus. Determining the relationship between the effective lens position and the actual position of a scleralfixated IOL will help to improve and refine the IOL calculation formulas for children with insufficient capsular support in the future.

Only two variables (AL and keratometry) are used to predict the postoperative IOL position in the thirdgeneration formulas which do not require measurement of ACD $(12,13,22)$. Consistent with previous studies, patients with inadequate capsular support, due to conditions such as congenital ectopia lentis, often have a flatter cornea $(4,11)$ and longer AL (10) than normal eyes; thus, using the lens constants from the ULIB website, which were acquired based on normal populations, will naturally reduce the accuracy of the third-generation formulas. In contrast, a preoperative $\mathrm{ACD}$ is required in the onward formulas including Barrett II and Haigis $(23,24)$. As reported in a previous study (25), it is not surprising that formulas that use more than two variables can help to acquire good outcomes for routine cataract surgery. However, compared to normal eyes, the measurement of ACD is usually inaccurate or is not applicable for eyes with severely dislocated lenses; therefore, using the value of ACD may reduce the accuracy of the corresponding formulas.

In this study, all formulas individually resulted in $<70 \%$ of eyes within $\pm 1.00 \mathrm{D}$ of the PE. These predicted outcomes using the five different formulas in our study were poorer than those in previous studies of common cataract populations that underwent routine cataract surgery, which suggested $>90 \%$ of patients should achieve postoperative spherical equivalents within $\pm 1.00 \mathrm{D}$ of the target refraction (26-29). Also, the accuracy of IOL calculation formulas in determining the postoperative refraction in our study was 
consistent with the results of a previous study on secondary scleral-fixated IOL implantation, which showed that Haigis generally underperformed compared to Holladay 1 and SRK/T (15). However, our results differed from those of studies of routine cataract surgery that reported Haigis and Barrett II to have a worse predicted outcome than the third-generation formulas (23-27). Nevertheless, after constants were optimized, five formulas resulted in $>68 \%$ (with an increase of $7.3 \%$ to $13.6 \%$ ) within $\pm 1.00 \mathrm{D}$ of the PE. Our results suggested that surgeons may be able to reduce the postoperative $\mathrm{PE}$ for this subgroup of patients by optimizing the constant for each formula based on their previous clinical data.

Both $\mathrm{AL}$ and mean keratometry have been reported to have significant associations with postoperative PE for the third-generation and Haigis formulas in routine cataract surgery $(26,27,30-32)$. These associations lie in that a longer AL resulted in a hyperopic refraction with SRK/T, Holladay, Hoffer Q, and Haigis, while a flatter keratometry (K) reading tended to result in a myopic PE with Haigis and Hoffer Q and a hyperopic PE with SRK/T. In this study, both univariate and multivariate linear regression analyses showed that postoperative PE was significantly associated with $\mathrm{AL}$ and keratometry for each formula. In other words, a trend toward hyperopic PE was found in eyes with a longer AL, and a myopic prediction could be accounted for by a flatter mean keratometry for each formula. Nevertheless, the associations of postoperative PE with $\mathrm{AL}$ and keratometry were weakest for SRK/T than for the other formulas. Additionally, our result differed from that of a previous study which reported $\mathrm{PE}$ to have no significant association with AL or keratometry for Barrett II in neither univariate nor multivariate linear regression analysis (27).

Based on these results of our study, both AL and keratometry could have a significant effect on postoperative $\mathrm{PE}$ in patients with insufficient capsular support when the SRK/T, Holladay 1, Hoffer Q, Haigis, and Barrett II formulas are used; thus, the potential effects of AL and keratometry on PE should be considered together when using these formulas. The weakest association between postoperative PE with AL and keratometry when using SRK/T may account for it having the smallest mean and median AE, which represents a better performance. SRK/ $\mathrm{T}$ has been suggested as the best choice for eyes with a flatter keratometry or a longer AL. However, definitive conclusions must be carefully drawn due to the relatively small sample size of this study.

In addition to biometric measurements, the accuracy of IOL calculation formulas can also be lens-related and dependent on the surgeon; thus, each surgeon should optimize lens constants for IOL calculation based on the biometric measurements, type of lens, and IOL position from the limbus to achieve better results in the future.

Our study has several limitations. Firstly, patients with insufficient capsular support are not encountered very often in our clinical practice, and the sample size may not reflect the overall population of this subgroup. Nevertheless, the results of post-calculated statistical power showed that the sample size of 110 involved in our study could achieve high enough power to detect the smallest difference of predictive error among different groups. Secondly, tilt and decentration of sclera-sutured IOL was another concern compared to "in-the-bag" IOL implantation (33). Although none of the participants were reported to have significant IOL tilt using the slit lamp or ultrasound biomicroscopy (UBM) examination, detailed tilt and decentration degree could not be assessed in this study due to its retrospective nature. For these reasons, our conclusion may not be generalizable, and further research including larger sample sizes and better designs is required. However, our results suggest that the characteristics and performance of IOL calculation are significantly different from common populations undergoing routine cataract surgery.

\section{Conclusions}

In conclusion, the use of the constants from the ULIB website tended to result in a myopic PE in patients with sclera-sutured IOL positioned $2 \mathrm{~mm}$ posterior to the limbus. In general, the SRK/T and Holladay 1 formulas were superior to the other formulas, including Haigis and Barrett II, for the prediction of postoperative refraction in eyes with insufficient capsular support. The accuracy of these formulas could be improved by using optimized constants; however, the performances of the scleral fixation of an IOL using these formulas are still poorer than the outcomes of routine cataract surgery. Further studies to optimize these formulas or to develop better formulas to address this challenge are still urgently required.

\section{Acknowledgments}

Funding: This work was supported by National Natural Science Foundation of China $(81873673,81900841)$ and the Fundamental Research Funds of the State Key Laboratory of Ophthalmology. 


\section{Footnote}

Reporting Checklist: The authors have completed the STROBE reporting checklist. Available at http://dx.doi. org/10.21037/atm-20-3290

Data Sharing Statement: Available at http://dx.doi. org/10.21037/atm-20-3290

Conflicts of Interest: All authors have completed the ICMJE uniform disclosure form (available at http://dx.doi. org/10.21037/atm-20-3290). The authors have no conflicts of interest to declare.

Ethical Statement: The authors are accountable for all aspects of the work in ensuring that questions related to the accuracy or integrity of any part of the work are appropriately investigated and resolved. The study was conducted in accordance with the Declaration of Helsinki (as revised in 2013). The study was approved by ethical committee of Zhongshan Ophthalmic Center (NO.:2019KYPJ184) and individual consent for this retrospective analysis was waived.

Open Access Statement: This is an Open Access article distributed in accordance with the Creative Commons Attribution-NonCommercial-NoDerivs 4.0 International License (CC BY-NC-ND 4.0), which permits the noncommercial replication and distribution of the article with the strict proviso that no changes or edits are made and the original work is properly cited (including links to both the formal publication through the relevant DOI and the license). See: https://creativecommons.org/licenses/by-nc-nd/4.0/.

\section{References}

1. Tabatabaei A, Kiarudi MY, Ghassemi F, et al. Evaluation of posterior lens capsule by $20-\mathrm{MHz}$ ultrasound probe in traumatic cataract. Am J Ophthalmol 2012;153:51-4.

2. Judge DP, Dietz HC. Marfan's syndrome. Lancet 2005;366:1965-76.

3. Zheng D, Wan P, Liang J, et al. Comparison of clinical outcomes between iris-fixated anterior chamber intraocular lenses and scleral-fixated posterior chamber intraocular lenses in Marfan syndrome with lens subluxation. Clin Exp Ophthalmol 2012;40:268-74.

4. Konradsen TR, Zetterström C. A descriptive study of ocular characteristics in Marfan syndrome. Acta
Ophthalmol 2013;91:751-5.

5. Rezar-Dreindl S, Stifter E, Neumayer T, et al, SchmidtErfurth U. Visual outcome and surgical results in children with Marfan syndrome. Clin Exp Ophthalmol 2019;47:1138-45.

6. Young AL, Agrawal R, Yuen HK, et al. Options for Marfan's syndrome. J Pediatr Ophthalmol Strabismus 2001;38:261.

7. Logan NS, Gilmartin B, Marr JE, et al. Community-based study of the association of high myopia in children with ocular and systemic disease. Optom Vis Sci 2004;81:11-3.

8. Norrby $\mathrm{S}$. Sources of error in intraocular lens power calculation. J Cataract Refract Surg 2008;34:368-76.

9. Aristodemou P, Knox CNE, Sparrow JM, et al. Formula choice: Hoffer Q, Holladay 1, or SRK/T and refractive outcomes in 8108 eyes after cataract surgery with biometry by partial coherence interferometry. J Cataract Refract Surg 2011;37:63-71.

10. Zhang Y, Jin G, Cao Q, et al. Distribution of axial length in Chinese congenital ectopia lentis patients: a retrospective study. BMC Ophthalmol 2017;17:113.

11. Konradsen TR, Koivula A, Kugelberg M, et al. Corneal curvature, pachymetry, and endothelial cell density in Marfan syndrome. Acta Ophthalmol 2012;90:375-9.

12. Holladay JT, Prager TC, Chandler TY, et al. A three-part system for refining intraocular lens power calculations. J Cataract Refract Surg 1988;14:17-24.

13. Retzlaff JA, Sanders DR, Kraff MC. Development of the SRK/T intraocular lens implant power calculation formula. J Cataract Refract Surg 1990;16:333-40.

14. Hoffer KJ. The Hoffer Q formula: a comparison of theoretic and regression formulas. J Cataract Refract Surg 1993;19:700-12.

15. Botsford BW, Williams AM, Conner IP, et al. Scleral Fixation of Intraocular Lenses with Gore-Tex Suture: Refractive Outcomes and Comparison of Lens Power Formulas. Ophthalmol Retina 2019;3:468-72.

16. Hwang ES, Warren CC, Koenig SB. Flanged intrascleral intraocular lens fixation with a single needle. J Cataract Refract Surg 2018;44:1526-7.

17. Gao S, Qin T, Wang S, et al. Sulcus Fixation of Foldable Intraocular Lenses Guided by Ultrasound Biomicroscopy. J Ophthalmol 2015;2015:520418.

18. Li B, Zhang Y, Gao X. Simple Technique for Transscleral Fixation of a Foldable Posterior Chamber Intraocular Lens Using a Single Suture. J Invest Surg 2020;33:446-52 .

19. Sinha R, Bansal M, Sharma N, et al. Transscleral SutureFixated Versus Intrascleral Haptic-Fixated Intraocular 


\section{Page 8 of 8}

Lens: A Comparative Study. Eye Contact Lens 2017;43:389-93.

20. Mizuno Y, Sugimoto Y. A comparative study of transscleral suture-fixated and scleral-fixated intraocular lens implantation. Int Ophthalmol 2019;39:839-45.

21. Hu ZX, Lin H, Ye L, et al. Sutureless Intrascleral HapticHook Lens Implantation Using 25-Gauge Trocars. J Ophthalmol 2018;2018:9250425.

22. Sheard RM, Smith GT, Cooke DL. Improving the prediction accuracy of the SRK/T formula: the T2 formula. J Cataract Refract Surg 2010;36:1829-34.

23. Amro M, Chanbour W, Arej N, et al. Third- and fourthgeneration formulas for intraocular lens power calculation before and after phakic intraocular lens insertion in high myopia. J Cataract Refract Surg 2018;44:1321-5.

24. Gökce SE, De Oca I M, Cooke DL, et al. Accuracy of 8 intraocular lens calculation formulas in relation to anterior chamber depth in patients with normal axial lengths. J Cataract Refract Surg 2018;44:362-8.

25. Shajari M, Kolb CM, Petermann K, et al. Comparison of 9 modern intraocular lens power calculation formulas for a quadrifocal intraocular lens. J Cataract Refract Surg 2018;44:942-8.

26. Melles RB, Holladay JT, Chang WJ. Accuracy of Intraocular Lens Calculation Formulas. Ophthalmology 2018;125:169-78.

27. Wan KH, Lam TC, Yu MC, et al. Accuracy and precision

Cite this article as: Li Z, Lian Z, Young CA, Zhao J, Jin G, Zheng D. Accuracy of intraocular lens calculation formulas for eyes with insufficient capsular support. Ann Transl Med 2021;9(4):324. doi: 10.21037/atm-20-3290

\section{Li et al. IOL calculation for eyes with insufficient capsular support}

of intraocular lens calculations using the new HillRBF version 2.0 in eyes with high axial myopia. Am J Ophthalmol 2019;205:66-73.

28. Hahn U, Krummenauer F, Kölbl B, et al. Determination of valid benchmarks for outcome indicators in cataract surgery: a multicenter, prospective cohort trial. Ophthalmology 2011;118:2105-12.

29. Simon SS, Chee YE, Haddadin RI, et al. Achieving target refraction after cataract surgery. Ophthalmology 2014;121:440-4.

30. Liu J, Wang L, Chai F, et al. Comparison of intraocular lens power calculation formulas in Chinese eyes with axial myopia. J Cataract Refract Surg 2019;45:725-31.

31. MacLaren RE, Sagoo MS, Restori M, et al. Biometry accuracy using zero- and negative-powered intraocular lenses. J Cataract Refract Surg 2005;31:280-90.

32. Hill DC, Sudhakar S, Hill CS, et al. Intraoperative aberrometry versus preoperative biometry for intraocular lens power selection in axial myopia. J Cataract Refract Surg 2017;43:505-10.

33. Hayashi K, Hayashi H, Nakao F, et al. Intraocular lens tilt and decentration, anterior chamber depth, and refractive error after trans-scleral suture fixation surgery. Ophthalmology 1999;106:878-82.

(English Language Editors: J. Jones and J. Reynolds) 